4 controls (2 peripheral neuropathy, 1 limb girdle muscle dystrophy, and metabolic myopathy) underwent after rest CEUS (Esaote MyLab, linear probe 13-5 $\mathrm{MHz}$, Sonovue $\left(\right.$ ) a t a room temperature of $20^{\circ}$ of the vastus lateralis and medialis muscles. CEUS was performed by 2 ultrasonographists with an expertise in muscle US blinded to the clinical data of the patients. CEUS muscle signal was expressed on a 0-4 scale as described in J Rheumatol 2001; 28:1271 per each muscle group and the global score was divided by four. Creatine kinase (CK), manual muscle test (MTT) and MRI of the thigh muscles were performed within maximum one month from the CEUS. MMT was expressed using the 0-5 Medical Research Council scale; intermediate points were converted into decimals as detailed in Kendall FP et al, Muscle Testing \& Function: Testing and Function with Posture and Pain. 5th ed., Lippincott Williams \& Wilkins, 2005. MRI of the thigh muscles was considered positive if it showed muscle edema. Myositis was defined active if CK was raised above the reference range and/or MMT showed progressive worsening. Results were expressed as median (range). Between-group comparison was performed with Mann-Whitney test. Statistical analysis was performed with SPSS version 20. The study was approved by the Ethics Committee and all patients provided their written consent.

Results: Median (range) age was 38 (69) years in the myositis and 41 (45) years in the control group $(p=0.89)$. Disease duration in the myositis group was 60 (334) months. CEUS muscle score was 0.5 (3) in the myositis group and $2(3)$ in the control group $(p=0.99)$. In the myositis group, CEUS score did not differ between treated and untreated patients $(p=0.84)$. CK values were 361 (6442) in the myositis group and 363 (799) in the control group $(p=0.68)$. MMT was significantly lower in the myositis group [4.33 (2)] than in the control group $[4.94(0)](p=0.038)$. CEUS was $77 \%(47-0595 \%$ confidence interval) sensitive and $67 \%$ (9-99\% 95\% confidence interval) specific for a diagnosis of myositis. CEUS was positive in 10/13 patients and negative in $3 / 13$ with active myositis, while was negative in $2 / 3$ patients and positive in $1 / 3$ with inactive myositis. Statistically, CEUS did not discriminate between active and inactive myositis (Fisher's exact test $p=0.21$ ). All controls had a positive CEUS. No association was found between MRI edema and a positive CEUS (intraclass correlation coefficient $p=0.5$ ). No correlation was found between CEUS score, on the one hand, and CK levels or MMT, on the other (Spearman's rho $\mathrm{p}>0.05$ ).

Conclusion: CEUS has moderate sensitivity for a diagnosis of myositis, but does not discriminate between myositis and some of its common mimickers. Larger studies are required to better evaluate the role of CEUS in patients with myositis.

Disclosure of Interests: Nicolo Pipitone Consultant of: Received royalties from Uptodate.com

Investigator for the gevokizumab in myositis Servier study (2014), the sirukumab in GCA GSK study (2016), PI for the ToReMy AIFA funded (2017) study and for the FOREUM funded (2018) GCA study

, Speakers bureau: Guest speaker at UCB-sponsored meetings: (Immunology Summits, Prague, 2012, 2013 \& 2014, MACRO Meet the expert at the ACademy of RheumatOlogy, Bologna 13 - 14 April 2012, GRAPPA Workshop, Milan 29 January 2016 and Rome 30 November 2017), Fininvest (Catania 2016), Aim Group (Reggio Emilia 2018), I\&C (Cologna, 2018), Alfa-Wassermann sponsored meeting (Rhewind, Bologna, February 2016 and 2019)

, Pierluigi Macchioni: None declared, Gianluigi Bajocchi: None declared, Carlo Salvarani Grant/research support from: consulting and investigator fees from Abbvie, Pfizer, MSD, Roche, Celgene, Novartis, Consultant of: consulting and investigator fees from Abbvie, Pfizer, MSD, Roche, Celgene, Novartis DOI: 10.1136/annrheumdis-2020-eular.3292

\section{SAT0339 NERVOUS SYSTEM INVOLVEMENT IN SYSTEMIC SCLEROSIS: A COHORT STUDY}

T. Porel ${ }^{1}$, S. DE Almeida Chaves ${ }^{1}$, D. Adoue ${ }^{1}$, L. Astudillo ${ }^{1}$, D. Ribes ${ }^{2}$, G. Prévôt ${ }^{3}$, F. Gaches ${ }^{4}$, M. Michaud ${ }^{4}$, L. Alric ${ }^{1}$, L. Sailler ${ }^{1}$, G. Pugnet ${ }^{1} .{ }^{1} \mathrm{CHU}$ Toulouse, Service de Médecine Interne, Toulouse, France; ${ }^{2} \mathrm{CHU}$ Toulouse, Service de Néphrologie, Toulouse, France; ${ }^{3} \mathrm{CHU}$ Toulouse, Service de Pneumologie, Toulouse, France; ${ }^{4}$ Hôpital Joseph Ducuing, Toulouse, France

Background: Nervous system involvement is considered to be rare in systemic sclerosis (SSc). Its prevalence is highly variable in SSc cohort studies and its prognosis is not well established.

Objectives: To determine the frequency, clinical characteristics, associations, and outcomes of different types of peripheral nervous system (PNS) and central nervous system (CNS) disease in a cohort of systemic sclerosis patients.

Methods: We have carried out a retrospective observational study by systematically analyzing the medical records of patients diagnosed with SSc in Toulouse University Hospital and Ducuing Hospital, south west France. We included patients who met the following inclusion criteria: being over 18 years of age on diagnosis, meeting the ACR /EULAR 2013 classification criteria, being diagnosed after 01/01/1966 and before 31/12/2018, at least 12 months of follow-up. Patients were followed until 31/12/2019. Nervous system involvement associated with SSc was included when there was involvement on or after diagnosis and after exclusion of all other causes. Only symptomatic clinical involvement was included. Ischemic or hemorrhagic strokes were excluded. We calculated the incidence of CNS and/or PNS disease during the follow-up period per 1,000 person-years. Kaplan-Meier curves were plotted to determine the cumulative incidence of nervous system disease. We evaluated associated factors of CNS and/or PNS disease using multivariable Cox regression.

Results: Of 447 SSc patients, $79.8 \%$ were female, 68 (15\%) were diffuse cutaneous SSc, 342 (77\%) were limited cutaneous SSc and $37(8 \%)$ were sine scleroderma SSc. The mean \pm SD age at diagnosis was $52.9 \pm 14.3$ years.

During the study period, 82 (18\%) patients experienced a PNS disease, 29 (6\%) a CNS disease. The incidence was 28 per 1,000 patient-years of any nervous system disease, with 22 per 1,000 patient-years and 6 per 1,000 patient-years of PNS disease and CNS disease, respectively. The most frequent were carpal tunnel syndrome (63\%) and polyneuropathies (12\%) for PNS disease, and headache $(45 \%)$ and seizures (10\%) for CNS disease.

Three significant independent associated factors with PNS disease occurrence were identified using multivariable Cox regression: $\mathrm{BMl}>23.1 \mathrm{~kg} / \mathrm{m}^{2}(\mathrm{HR}=1.06$ [1.01-1.12]), joint involvement $(\mathrm{HR}=2.7[1.3-5.5])$, and an alteration in the left ventricular ejection fraction $(\mathrm{HR}=3.8[1.4-10.3])$.

Four significant independent associated factors with CNS disease occurrence were identified: age $>54$ years $(\mathrm{HR}=2.5$ [1.1-6.0]), positive anti-PmScl testing $(\mathrm{HR}=6.4$ [1.5-28.2]), Caucasian origin $(\mathrm{HR}=0.2[0.1-0.5])$ and hemoglobin $12 \mathrm{~g} / \mathrm{dl}(\mathrm{HR}=0.2[0.04-0.8])$.

Nervous system disease occurrence did not appear to have a negative impact on the survival of SSc patients (log-rank $p=0.56$ ).

Conclusion: This study shows that specific nervous system disease in SSc is not uncommon and does not appear to increase mortality, but it could have an impact on functional prognosis and needs to be monitored.

Disclosure of Interest: None declared

DOI: 10.1136/annrheumdis-2020-eular.6142

\section{SAT0340 A REDUCED NUMBER OF CAPILLARIES AND AN INCREASED NUMBER OF MEGACAPILLARIES PREDICT THE DEVELOPMENT OF SYSTEMIC SCLEROSIS IN RAYNAUD'S PHENOMENON PATIENTS AT RISK}

A. Riccardi ${ }^{1}$, A. Marcoccia ${ }^{2}$, S. Fasano ${ }^{1}$, T. Guastafierro ${ }^{2}$, R. Irace ${ }^{1}$, V. Messiniti ${ }^{1}$, F. Bondanini ${ }^{2}$, A. Sanduzzi ${ }^{3}$, M. Bocchino ${ }^{3}$, A. Ciani ${ }^{2}$, M. D'alto $^{4}$, P. Argiento ${ }^{4}$, G. M. De Matteis ${ }^{2}$, A. Spanò ${ }^{2}$, G. Valentini ${ }^{1}$. ${ }^{1}$ University of Campania "Luigi Vanvitelli", Department of Precision Medicine, Naples, Italy; ${ }^{2}$ Sandro Pertini Hospital, Centro di Riferimento Interdisciplinare, Interdipartimentale per la Diagnosi Precoce della Sclerodermia, Rome, Italy; ${ }^{3}$ University Federico II, Department of Clinical and Experimental Medicine, Naples, Italy; ${ }^{4}$ University of Campania “Luigi Vanvitelli”, Department of Cardiology, Naples, Italy

Background: Undifferentiated connective tissue disease at risk for systemic sclerosis (UCTD-risk-SSc) is a condition characterised by Raynaud's phenomenon and either SSc marker autoantibodies or typical capillaroscopic findings or both, unsatisfying classification criteria for SSc and evolving into definite SSc in about $30-50 \%$ of cases $(1,2)$. Recently, we developed a weighted score based on a baseline IF-ANA titer $\geq 1: 320$, marker autoantibody positivity and presence of avascular areas at videocapillaroscopy identifying patients who will evolve with a $91.3 \%$ sensitivity and a $73.2 \%$ specificity (3).

Objectives: To improve the predictivity of the score assessing the role of marker autoantibody ELISA titer and further capillaroscopic items.

Methods: The 102 UCTD-risk-SSc patients investigated for the development of the previous score were reassessed for anti-Scl-70 and anti-centromere antibody titers detected by ELISA and for the mean number of capillaries observed in the same capillaroscopic field (Cs) and the total number of giant capillaries (GC) by videocapillaroscopy (4). Each patient was evaluated every 6 months to assess disease progression. Risk prediction was assessed by Cox regression analyses. The predictive value of the score was determined by receiver operating curve (ROC) analysis.

Results: Table 1 shows the resulting predictive variables in multivariate Cox analysis and their relative weight in a 10-point scale. No increase in the predictivity was detected by adding the anti-Scl-70 and anti-centromere antibody ELISA titers. However, a mean number of $\mathrm{C} s \leq 5 / \mathrm{mm}$ and $\mathrm{GC}>5$ improved the score. At ROC analysis (Figure 1) a score $>3.25$ predicted evolution to SSc with a sensitivity of $93.5 \%$ and a $75 \%$ specificity $(A \cup C=0.91)$. 